\title{
Construção de um instrumento para apoio a aplicação do processo de enfermagem em infecções sexualmente transmissíveis: Nota prévia
}

\author{
Construction of an instrument to support the application of the nursing process in sexually \\ transmitted infections: Previous note \\ Construcción de un instrumento para apoyar la aplicación del proceso de enfermería en infecciones \\ de transmisión sexual: Nota previa
}

\begin{abstract}
Resumo
O estudo tem por objetivo construir um instrumento para apoio ao processo de enfermagem em Infecções Sexualmente Transmissíveis. Trata-se de um estudo quantitativo e transversal do tipo metodológico organizado em cinco etapas: identificação das evidências empíricas, seleção das variáveis relevantes para anamnese e exame físico, elaboração dos diagnósticos, resultados e intervenções de enfermagem, construção da versão inicial do instrumento e validação de conteúdo do instrumento. A construção do instrumento fundamentou-se na Teoria das Necessidades Humanas Básicas e o material empírico foi o Protocolo Clínico e Diretrizes Terapêuticas para Atenção Integral às Pessoas com Infecções Sexualmente Transmissíveis. Para a proposição dos diagnósticos, resultados e intervenções utilizou-se a Classificação Internacional para a Prática de Enfermagem. Complementarmente, elaborou-se a correspondência de acordo com a Classificação Internacional de Assistência Primária, tendo em vista sua aplicação na atenção primária durante a consulta de enfermagem. O instrumento contempla quesitos relevantes para a consulta de enfermagem, abrangendo a sexualidade, alterações geniturinárias, testagem, imunização e aconselhamento para IST. Espera-se promover a qualificação da atenção em IST a partir da utilização de um instrumento de coleta de dados durante a consulta de enfermagem, especificamente para pacientes afetados por IST assintomáticas, a fim de nortear as ações a serem executadas pelo profissional conforme preconização do protocolo clínico nacional.
\end{abstract}

Palavras-chave: Doenças Sexualmente Transmissíveis; Processo de enfermagem; Teoria de enfermagem.

\begin{abstract}
The study aims to build an instrument to support the nursing process in Sexually Transmitted Infections. This is a quantitative and transversal study of a methodological type organized in five stages: identification of empirical evidence, selection of relevant variables for anamnesis and physical examination, preparation of diagnoses, results and nursing interventions, construction of the initial version of the instrument and validation content of the instrument. The construction of the instrument was based on the Theory of Basic Human Needs and the empirical material was the Clinical Protocol and Therapeutic Guidelines for Comprehensive Care for People with Sexually Transmitted Infections. For the proposition of diagnoses, results and interventions, the International Classification for Nursing Practice was used. In addition, correspondence was prepared in accordance with the International Classification of Primary Care, with a view to its application in primary care during the nursing consultation. The instrument includes relevant items
\end{abstract}


for the nursing consultation, covering sexuality, genitourinary changes, testing, immunization and STI counseling. It is expected to promote the qualification of care in STIs from the use of a data collection instrument during the nursing consultation, specifically for patients affected by asymptomatic STIs, in order to guide the actions to be performed by the professional as recommended by the protocol national clinician.

Keywords: Sexually Transmitted Diseases; Nursing process; Nursing theory.

\section{Resumen}

El estudio tiene como objetivo construir un instrumento de apoyo al proceso de enfermería en Infecciones de Transmisión Sexual. Se trata de un estudio cuantitativo y transversal de tipo metodológico organizado en cinco etapas: identificación de evidencia empírica, selección de variables relevantes para anamnesis y exploración física, elaboración de diagnósticos, resultados e intervenciones de enfermería, construcción de la versión inicial del instrumento y validación contenido del instrumento. La construcción del instrumento se basó en la Teoría de las Necesidades Humanas Básicas y el material empírico fue el Protocolo Clínico y Lineamientos Terapéuticos para la Atención Integral de Personas con Infecciones de Transmisión Sexual. Para la propuesta de diagnósticos, resultados e intervenciones se utilizó la Clasificación Internacional para la Práctica de Enfermería. Además, se elaboró correspondencia de acuerdo con la Clasificación Internacional de Atención Primaria, con miras a su aplicación en atención primaria durante la consulta de enfermería. El instrumento incluye ítems relevantes para la consulta de enfermería, que cubren sexualidad, cambios genitourinarios, pruebas, inmunizaciones y consejería de ITS. Se espera promover la calificación de la atención de ITS a partir del uso de un instrumento de recolección de datos durante la consulta de enfermería, específicamente para pacientes afectados por ITS asintomáticas, con el fin de orientar las acciones a realizar por el profesional según lo recomendado por el protocolo clínico nacional.

Palabras clave: Enfermedades de Transmisión Sexual; Proceso de enfermería; Teoría de enfermería.

\section{Introdução}

As Infecções Sexualmente Transmissíveis (IST) configuram-se como um agravo à saúde de âmbito global, uma vez que provocam malefícios a curto e longo prazo, que repercutem nas relações interpessoais da população acometida. Estima-se que, mundialmente, a cada ano, 357 milhões de pessoas adoecem com uma das quatro IST mais frequentes: clamídia, gonorreia, sífilis e tricomoníase (Who, 2019). No Brasil, não há notificação compulsória para todas as IST, dificultando a visibilidade epidemiológica do problema. Contudo, as estimativas apontam que, a cada ano, ocorrem 1.541.800 casos de gonorreia, 1.967.200 de clamídia, 685.400 de Papilomavírus Humano (HPV), 640.900 de herpes genital e 937.000 casos de sífilis (Brasil, 2020).

O controle das IST exige esforços por parte dos profissionais de saúde, pois os mesmos são responsáveis por prevenir e evitar agravos à saúde severos oriundos dessas infecções, como infertilidade, complicações na gravidez e no parto e morte fetal (Brasil, 2019). Para tanto, a atenção em IST está relacionada à prática de intervenções baseadas em evidências, implementação das estratégias de rastreio, diagnóstico adequado e tratamento oportuno das infecções sintomáticas e assintomáticas (Who, 2016).

Para o desempenho das ações de controle das IST, destacam-se os profissionais atuantes na Atenção Primária à Saúde (APS), especialmente os enfermeiros, que desempenham a função de garantir o acolhimento, orientação e tratamento adequados ao paciente, bem como, são responsáveis pelo encaminhamento aos demais serviços de saúde que integram a média e alta complexidade, quando necessário (Brasil, 2015). Além disso, o enfermeiro é responsável pela consulta de enfermagem, solicitação de exames, prescrição de medicamentos, atividades em grupo, aconselhamento, oferta de testes e vacinas de acordo com os protocolos clínicos nacionais estabelecidos pelo Ministério da Saúde (Brasil, 2020).

Nesse entendimento, o desempenho das competências do enfermeiro para o controle das IST é delimitado durante a formação e reafirmado por meio das diretrizes e protocolos para prevenção e abordagem dos casos. Contudo, estudos que avaliaram a qualidade da assistência de enfermagem em IST identificaram que os profissionais não realizavam as condutas preconizadas em protocolo clínico nacional. Esses mesmos estudos evidenciaram que o levantamento de informações na anamnese ocorreu de maneira incipiente, enquanto o exame físico, a oferta de testes, o tratamento e o aconselhamento foram realizados de maneira incompleta ou não eram realizados (Ferreira, et al., 2018; Santos, et al., 2019).

Diante disso, verificou-se a necessidade de aprimoramento da qualidade da assistência prestada em IST para realização do cuidado de enfermagem fundamentado no pensamento crítico, sistemático e clínico. Para tanto, enfatiza-se a necessidade que 
o enfermeiro desenvolva o cuidado em IST por meio do Processo de Enfermagem (PE), conforme preconiza a legislação da profissão (Cofen, 2009).

O PE é intrínseco à assistência de enfermagem e pode potencializar as ações do enfermeiro na APS, especificamente na atenção em IST. Desse modo, verifica-se a relevância da aplicação do PE nos serviços, que é operacionalizada por meio de execução das etapas e pelo emprego de instrumentos estruturados de investigação que auxiliem o profissional na realização do cuidado sistematizado.

Para a organização do PE são previstas cinco etapas inter-relacionadas, interdependentes e recorrentes: Investigação (coleta de dados e exame físico); Diagnóstico de enfermagem; Planejamento (resultados esperados); Implementação da assistência de enfermagem e Avaliação (Filgueiras, et al., 2019). Essas etapas, desenvolvidas com base em evidências científicas, teorias de enfermagem e em linguagem padronizada, são viabilizadas por meio de instrumentos que direcionam a coleta de dados, o registro de informações e as ações do enfermeiro. Entretanto, observa-se que os instrumentos utilizados na prática de enfermagem restringem-se ao levantamento de dados sociodemográficos e informações sobre o exame físico de forma genérica, fundamentados no modelo biomédico (Tolentino, et al., 2018).

Especificamente na atenção em IST, verificou-se em nossa prática assistencial a ausência de instrumento sistematizado para apoio ao processo de enfermagem. Do mesmo modo, a literatura consultada demonstra a escassez de estudos sobre o tema. Assim, torna-se imperativo o investimento em pesquisas que contribuam para o desenvolvimento de tecnologias do cuidado em enfermagem, especialmente por meio da construção de instrumentos que viabilizem a obtenção de dados de forma sistemática e segura. Acredita-se que o investimento em ferramentas tecnológicas é capaz de aumentar a eficiência do cuidado em enfermagem e atender às necessidades em saúde (Koerich, et al., 2006; Souza Neto, 2017), pois envolve o raciocínio teórico e prático sistematizado que permite a construção de meios que organizem o PE e qualificam a assistência.

Desse modo, o presente estudo possui como objetivo construir um instrumento para apoio ao processo de enfermagem em Infecções Sexualmente Transmissíveis voltado ao público adulto e abrangendo pacientes de gêneros e orientações sexuais diversas.

\section{Metodologia}

O estudo está inserido no Programa Institucional de Bolsas de Iniciação Científica (PIBIC), vinculado ao Conselho Nacional de Desenvolvimento Científico e Tecnológico (CNPq) e possui delineamento quantitativo e transversal do tipo metodológico.

A pesquisa de cunho quantitativo lida com fatos, portanto, as variáveis são rigorosamente determinadas e sua mensuração é pressuposta pelo próprio método. A análise dos dados é mediada por um critério matemático utilizado para validação dos resultados obtidos na pesquisa (Polit, Beck \& Hungler, 2004). Quanto à temporalidade, o estudo caracteriza-se como transversal por avaliar diferentes variáveis em um único espaço de tempo (Carvalho, et. al., 2019).

Esclarece-se que os estudos metodológicos possibilitam a investigação de métodos para coleta e organização de dados, favorecendo a condução da investigação com rigor acentuado, visto que o levantamento de informações pertinentes possibilitará a tomada de decisão e a aplicação de intervenções seguras e eficazes no problema apresentado (Pereira, et al., 2018). Em enfermagem esse tipo de estudo permite a organização de informações para o desenvolvimento de instrumentos para o cuidado.

Para construção do instrumento foram elencadas etapas de desenvolvimento, descritas a seguir: 1) estabelecimento da estrutura conceitual, definição dos objetivos e da população envolvida; 2) construção dos itens e domínios; 3) elaboração dos diagnósticos, resultados e intervenções de enfermagem 4) estruturação da versão inicial do instrumento; 5) validação de conteúdo (Milani, Alexandre \& Coluci, 2013). 
A primeira etapa, estabelecimento da estrutura conceitual, definição dos objetivos e da população envolvida, foi desenvolvida por meio da análise minuciosa do Protocolo Clínico e Diretrizes Terapêuticas para Atenção Integral às Pessoas com Infecções Sexualmente Transmissíveis com a finalidade de justificar a relevância da criação de um novo instrumento, definir o contexto em que será utilizado e comprovar sua utilidade e importância para a assistência em saúde (PCDT-IST) (Brasil, 2020).

A segunda etapa, construção dos itens e domínios, consistiu na busca na literatura de instrumentos já existentes que abordassem o âmbito de IST, bem como, das especificações contidas no PCDT-IST. Após essa seleção, foram incluídos os grupos prioritários, orientações de prevenção, detecção, testagem e imunização, sinais e sintomas mais frequentes desencadeados por IST sintomáticas e excluídos os ítens que foram considerados ambíguos, incompreensíveis, com termos vagos ou em duplicidade. Como arcabouço teórico, utilizou-se a Teoria das Necessidade Humanas Básicas (Horta, 1979), considerando as necessidades psicobiológicas, psicoespirituais e psicossociais. Os itens e domínios selecionados foram organizados de acordo com cada necessidade e dispostos em um quadro.

$\mathrm{Na}$ terceira etapa, foi realizada a elaboração dos diagnósticos, resultados e intervenções de enfermagem de acordo com a Classificação Internacional para a Prática de Enfermagem - CIPE (Garcia, 2020). Desse modo, foi elaborado um quadro com propostas para os possíveis diagnósticos, resultados e intervenções encontrados que fossem correspondentes às IST e que, consequentemente, serão avaliados em etapa posterior. Adicionalmente, foi utilizada a Classificação Internacional de Assistência Primária (CIAP-2) (Brasil, 2010), tendo em vista sua aplicação no contexto da APS durante o cuidado em enfermagem.

A quarta etapa, estruturação da versão inicial do instrumento, consolidou as etapas anteriores, bem como, foram organizados os itens em seus respectivos domínios e estabelecido o formato geral do instrumento. Tal etapa foi desenvolvida, concomitantemente, à terceira etapa. O instrumento, embasado nos resultados das etapas anteriores, foi norteado pela Teoria das Necessidade Humanas Básicas e apresentado nos resultados esperados. A estrutura e a sequência do instrumento seguiram uma ordem lógica, a fim de manter a objetividade e clareza para o profissional e para os pacientes.

A quinta e última etapa, validação de conteúdo, está sendo realizada no momento através da submissão do instrumento em sua versão inicial a um grupo de juízes especialistas, os quais avaliarão o instrumento de forma criteriosa.

Esclarece-se que a pesquisa foi submetida e aprovada pelo Comitê de Ética e Pesquisa do Hospital Universitário Alcides Carneiro (HUAC), parecer $n^{\circ} 4.568 .582$.

\section{Resultados e Discussão}

\subsection{Identificação das evidências empíricas}

Para construção do instrumento de apoio ao processo de enfermagem tornou-se necessária a busca e a análise das evidências científicas direcionadas aos profissionais de saúde no âmbito das IST. Para tanto, utilizou-se o Protocolo Clínico e Diretrizes Terapêuticas para Atenção Integral às Pessoas com Infecções Sexualmente Transmissíveis (PCDT), documento elaborado pelo Ministério da Saúde, em sua versão 2020, sendo considerados os critérios de prevenção, detecção precoce, diagnóstico e tratamento contidos no mesmo. Realizou-se a seleção e retirada dos dados para o presente instrumento a partir da utilização de critérios de inclusão e exclusão. Foram incluídos os cinco grupos de IST Sintomáticas designados no protocolo clínico nacional, os sinais e sintomas mais frequentes encontrados no paciente acometido, recomendações de testagem e imunização, diagnósticos e intervenções necessárias para o público adulto de todos os gêneros e orientações sexuais e a população chave e prioritária para IST. Foram excluídos os grupos de IST consideradas assintomáticas e as intervenções destinadas ao público adolescente (PDCT-IST) (Brasil, 2020).

O PCDT-IST reúne estudos atualizados, baseados em evidências científicas, que estabelecem critérios para identificação, diagnóstico de infecções, tratamento, ações de prevenção e o acompanhamento dos casos. Todas as orientações 
recomendadas devem ser seguidas pelos profissionais de saúde e gestores do Sistema Único de Saúde - SUS, visto que o PCDTIST considera critérios de eficácia, segurança, efetividade e custo-efetividade (Brasil, 2020).

As variáveis selecionadas foram organizadas de acordo com a Teoria das Necessidades Humanas Básicas (Horta, 1979), conforme descrito a seguir.

\subsection{Organização das variáveis de anamnese e exame físico}

O modelo mais conhecido e utilizado no Brasil para a implementação do PE foi o estabelecido por Horta (1979), intitulado Teoria das Necessidades Humanas Básicas. Tal teoria de enfermagem subsidia e dispõe de itens mínimos indispensáveis que compõem a primeira etapa do PE: investigação. Desse modo, o presente instrumento pautado na ótica desta teórica, buscou incluir na anamnese e exame físico as informações indispensáveis pensados por Horta, a fim resultar em uma investigação completa, que possua dados suficientes para o estabelecimento de um PE viável para a tomada de decisão eficiente e eficaz ao cliente (Horta, 1979; Almeida, et al, 2018).

A anamnese é definida como a primeira fase do processo de enfermagem, contendo dados subjetivos, objetivos, históricos e atuais. Pensando nisso, ao concluir a anamnese o profissional encontra-se habilitado para oferecer um quadro completo da história da doença atual e pregressa do cliente. Estes dados podem ser obtidos por meio de uma consulta norteada por um instrumento de coleta de dados, facilitando a organização das informações e a condução da anamnese (Santos, Veiga \& Andrade, 2011).

O exame físico busca interpretar sinais e sintomas observando pontos relevantes e anormalidades através das técnicas de inspeção, palpação, percussão e ausculta. O exame físico permite ao enfermeiro validar os achados da anamnese, identificar problemas, definir diagnóstico de enfermagem, planejar e programar intervenções de enfermagem e acompanhar a evolução do paciente a fim de estabelecer os resultados esperados (Potter \& Perry, 2013).

Para o presente estudo as questões que compõem a anamnese corresponderam à identificação, dados socioeconômicos, queixa principal, antecedentes pessoais, população chave e prioritária para IST, imunização e testagem para IST e história pregressa do problema atual. As questões que compõem o exame físico correspondem às Necessidades Humanas Básicas (Necessidades Psicobiológicas, Psicossociais e Psicoespirituais), as quais estão dispostas detalhadamente no Quadro I a seguir:

Quadro 1 - Variáveis para anamnese e exame físico segundo a teoria das NHB, Campina Grande, 2021.

\begin{tabular}{|c|c|c|}
\hline Necessidades Psicobiológicas & Necessidades Psicossociais & Necessidades Psicoespirituais \\
\hline  & 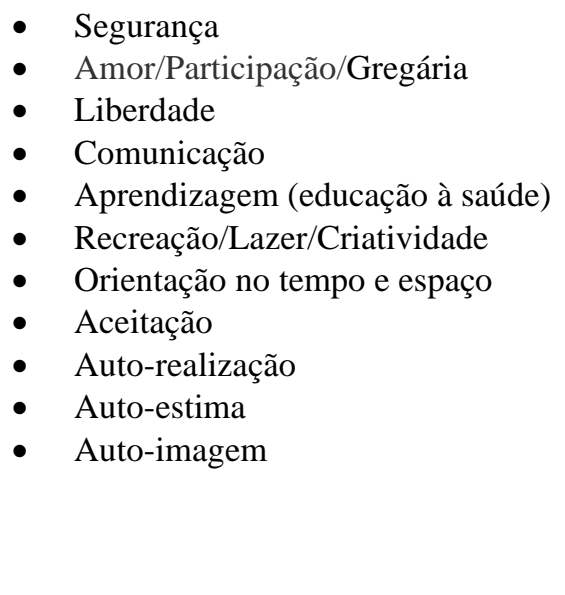 & $\begin{array}{l}\text { Religiosa ou teológica, } \\
\text { ética ou de filosofia de } \\
\text { vida }\end{array}$ \\
\hline
\end{tabular}

Fonte: Autores (2021), adaptado de Horta (1979). 
A abordagem do instrumento é voltada para uma clientela portadora de IST, com queixas relacionadas às IST e/ou pacientes que se enquadram nas características chave e de vulnerabilidade para IST (Brasil, 2020). Para tanto, a disposição das necessidades teve como foco os problemas mais frequentes e prevalentes encontrados nessa clientela, buscando objetividade e resolutividade.

Tornou-se necessário realizar uma adaptação da teoria original, acrescentando quesitos relevantes no que tange à temática de sexualidade e IST, bem como a inclusão de seções específicas ao público-alvo proposto, cabendo destacar a inclusão do exame físico geral, exame genital masculino e feminino, testagem, imunização e aconselhamento, a fim de tornar o processo de enfermagem completo, conforme preconizado no protocolo clínico nacional.

\subsection{Seleção dos diagnósticos e resultados de Enfermagem}

Com base na teoria das NHB, foram selecionados os possíveis diagnósticos e resultados para os dados elencados na etapa de investigação, anamnese e exame físico. Em nosso estudo, os diagnósticos foram propostos para cada um dos principais grupos de IST Sintomáticas, sendo: Corrimento vaginal, Corrimento uretral, Úlcera genital, Doença Inflamatória Pélvica (DIP) e Verrugas Anogenitais. Esta escolha justifica-se pelo fato de que no Brasil, devido às dificuldades para o diagnóstico etiológico, propõe-se o agrupamento das IST mais frequentes, possibilitando melhor identificação e tratamento (Brasil, 2020). Desse modo, optou-se por propor os diagnósticos de enfermagem para esses grupos de doenças, considerando-se as informações contidas na etapa de investigação, que quando coletadas indicarão as necessidades afetadas e poderão convergir para o conjunto de infecções mais prevalentes no Brasil.

Os diagnósticos e resultados de enfermagem são essenciais para a implementação do PE, uma vez que a assistência se torna sistematizada, traz benefícios tanto para o cliente quanto para o profissional (Viana \& Pires, 2014). Desse modo, a partir dos possíveis grupos de IST sintomáticas estabelecidos, foram elaborados os diagnósticos e os resultados de enfermagem, bem como a correspondência CIAP-2, os quais se encontram compilados e organizados no Quadro 2. 
Quadro 2 - Diagnósticos e resultados CIPE e correspondência CIAP-2 para cada grupo de IST Sintomática. Campina Grande, 2021.

\begin{tabular}{|c|c|c|c|}
\hline Grupo de IST & Diagnósticos de enfermagem & Resultados & $\begin{array}{l}\text { Correspondência } \\
\text { CIAP-2 }\end{array}$ \\
\hline $\begin{array}{l}\text { - Corrimento } \\
\text { Vaginal }\end{array}$ & $\begin{array}{ll}\text { - } & \text { Candidíase presente } \\
\text { - } & \text { Prurido leve em região } \\
& \text { vulvar } \\
\text { - } & \text { Dispareunia moderada } \\
\text { - } & \text { Inflamação moderada } \\
\text { - } & \text { Des região vulvar } \\
& \text { Vaginal, Excessiva }\end{array}$ & $\begin{array}{ll}\text { - } & \text { Candidíase ausente } \\
\text { - } & \text { Prurido melhorado } \\
\text { em região vulvar } \\
\text { - } & \text { Dispareunia ausente } \\
\text { - } & \text { Inflamação } \\
& \text { melhorada em região } \\
\text { vulvar }\end{array}$ & $\begin{array}{ll}\text { - } & \text { Candidíase } \\
\text { - } & \text { Tenital feminina } \\
\text { Tricomoniase } \\
\text { - } \quad \text { Venital feminina } \\
\text { Vaginite/Vulvite }\end{array}$ \\
\hline $\begin{array}{l}\text { - Corrimento } \\
\text { Uretral }\end{array}$ & $\begin{array}{ll}\text { - } & \text { Disúria presente } \\
\text { - } & \text { Infecção moderada em } \\
\text { prepúcio e uretra } \\
\text { - } & \text { Eritema presente em } \\
& \text { região de prepúcio }\end{array}$ & $\begin{array}{ll}\text { - } & \text { Disúria ausente } \\
\text { - Infecção melhorada } \\
\text { - } & \text { Secreçãáno normal }\end{array}$ & - Balanite \\
\hline $\begin{array}{l}\text { - Úlcera } \\
\text { Genital }\end{array}$ & $\begin{array}{l}\text { - Úlcera presente em } \\
\text { região pubiana } \\
\text { - Eczema }\end{array}$ & $\begin{array}{l}\text { - Úlcera ausente em } \\
\text { região pubiana }\end{array}$ & - Herpes genital \\
\hline $\begin{array}{l}\text { - Doença } \\
\text { Inflamatóri } \\
\text { a Pélvica } \\
\text { (DIP) }\end{array}$ & $\begin{array}{ll}\text { - } & \text { Dor abdominal } \\
\text { moderada } \\
\text { - } & \text { Dispareunia moderada } \\
\text { - } & \text { Massa pélvica } \\
\text { - } & \text { Sangramento vaginal } \\
\text { anormal }\end{array}$ & $\begin{array}{ll}\text { - } & \text { Dor abdominal } \\
\text { ausente } \\
\text { - } & \text { Dispareunia ausente } \\
\text { - } & \text { Sangramento vaginal } \\
\text { nos níveis de } \\
\text { normalidade }\end{array}$ & $\begin{array}{l}\text { - Doença } \\
\text { Inflamatória } \\
\text { Pélvica } \\
\text { - Doença colo }\end{array}$ \\
\hline $\begin{array}{ll}- & \text { Verrugas } \\
\text { Anogenitai } \\
\mathrm{s}\end{array}$ & $\begin{array}{l}\text { - Verruga presente em } \\
\text { região pubiana }\end{array}$ & $\begin{array}{l}\text { - Verruga ausente em } \\
\text { região pubiana }\end{array}$ & $\begin{array}{l}\text { - Condiloma } \\
\text { acuminado }\end{array}$ \\
\hline
\end{tabular}

Fonte: Autores (2021).

Para elaboração dos diagnósticos e resultados de Enfermagem, utilizamos a Classificação Internacional para a Prática de Enfermagem (CIPE®), que corresponde a uma terminologia padronizada, ampla e complexa, inserida no domínio da prática de enfermagem mundialmente. Sua utilização proporciona a coleta, o armazenamento e a análise de dados de enfermagem em diversos idiomas e regiões geográficas, contribuindo para melhorar a atenção à clientela e facilitar a comunicação dos enfermeiros entre si e com outros profissionais de saúde (Cipe, 2020). Ademais, a escolha pela CIPE® baseou-se nos benefícios trazidos por sua implementação, tais como facilitar o raciocínio clínico e a documentação padronizada do cuidado prestado ao paciente pelo profissional de enfermagem em sistemas manuais de registros, como é o caso do instrumento de coleta de dados, bem como, por representar o domínio da prática de enfermagem a nível mundial (Truppell, et. al., 2009; Cipe, 2020).

Complementarmente, foi utilizada a Classificação Internacional da Atenção Primária (CIAP-2) para composição do instrumento, que corresponde a uma ferramenta adequada à Atenção Básica, que permite identificar os problemas diagnosticados pelos profissionais de saúde e os motivos da consulta seguindo a sistematização Subjetivo, Objetivo, Avaliação e Plano (SOAP) prevista no Prontuário Eletrônico do paciente. Salienta-se a relevância da articulação entre a CIAP-2 e uma classificação de enfermagem, tendo em vista que o primeiro é um item de preenchimento obrigatório no Sistema de Informação em Saúde da 
Atenção Básica (e-SUS/SIS-AB), mas que não substitui o Diagnóstico de Enfermagem (Brasil, 2016; Ribeiro \& Padoveze, 2018) na APS.

Salienta-se que a implementação do PE acontece de forma dinâmica ao decorrer da realização do cuidado de enfermagem e depende da particularidade de cada paciente atendido (Barros, et al., 2015). Sendo assim, compreende-se que as IST afetam as NHB de forma distinta em cada cliente, cabendo ao profissional que utilizará o instrumento avaliar e sugerir outros diagnósticos e condutas que o mesmo considere eficaz para cada caso individualmente.

Reconhece-se que em um processo de adoecimento por IST os três âmbitos de NHB podem ser afetados, ultrapassando o limite dos sinais e sintomas patológicos e comprometendo a vida diária, relacionamentos afetivos e satisfação sexual dos pacientes (Fernandes \& Narchi, 2013). Entretanto, os diagnósticos e resultados de enfermagem presentes no Quadro 2 limitaramse à patologia, abrangendo as principais manifestações das IST.

\subsection{Seleção das intervenções de Enfermagem}

Ao finalizar a elaboração dos diagnósticos de enfermagem, o profissional realizará o planejamento da assistência, traçará os resultados esperados e, por fim, as intervenções que envolvem o aconselhamento, tratamento, orientação, notificação, vacinação e imunização, que devem ser escolhidas de acordo com cada cliente atendido (Brasil, 2020). Essas intervenções de enfermagem foram organizadas e dispostas no Quadro 3.

Quadro 3 - Intervenções CIPE e correspondência CIAP-2 para pessoas com Infecção Sexualmente Transmissíveis Sintomáticas. Campina Grande, 2021.

Intervenções CIPE

- Oferecer vacinação para hepatite A e hepatite B, e para HPV, quando indicado.

- Ofertar e realizar aconselhamento e teste rápido para HIV, hepatites virais e sífilis

- Tratar, acompanhar e orientar a pessoa e suas parcerias sexuais.

- Prescrever medicação

- Promover Adesão à Medicação

- Orientar sobre Comportamento Sexual

- Orientar sobre Higiene Vaginal

- Orientar sobre Prevenção de Infecção Cruzada

- Agendar Consulta de Acompanhamento (ou Consulta Subsequente)

- Notificar o caso, quando indicado
Correspondência CIAP-2

- Vacinação

- Educação em saúde/Aconselhamento

- Consulta com especialista

- $\quad$ Prescrição de medicação

- Escuta terapêutica

Fonte: Autores (2021).

As intervenções escolhidas para compor o instrumento são essenciais ao cuidado relativo aos cinco grupos de IST sintomáticas e devem ser oferecidas a todo paciente acometido, uma vez que todas as ações giram em torno da promoção e prevenção da saúde do indivíduo de acordo com o PCDT-IST. Dentre as ações fundamentais no controle e combate às IST, estão: oferta de testes, oferta de vacinação, promoção de acompanhamento adequado, notificação de casos indicados e aconselhamento quanto à educação sexual (Brasil, 2020).

O profissional de saúde possui total autonomia para acrescentar outras intervenções a depender dos dados coletados durante a etapa de anamnese e exame físico do atendimento, ressaltando a individualidade de cada paciente, não se limitando apenas às intervenções dispostas no presente instrumento. 
3.5 Versão inicial do instrumento e processo de validação

Após a realização das etapas descritas foi elaborada a versão inicial do instrumento organizada nas seguintes seções: Identificação, Dados Socioeconômicos, Queixa principal, Antecedentes pessoais, População chave e prioritária para IST, Imunização e testagem para IST, História pregressa do problema atual, Necessidades Psicobiológicas, Necessidades Psicossociais, Necessidades Psicoespirituais, Diagnósticos de Enfermagem, Resultados de Enfermagem, Intervenções de Enfermagem, Evolução de Enfermagem e Avaliação de Enfermagem.

Na quinta e última etapa do estudo, denominada validação de conteúdo do instrumento, serão escolhidos avaliadores que tenham graduação em Enfermagem, conhecimento em PE e atuação na atenção em IST há pelo menos 1 ano. Após essa seleção, o instrumento será enviado aos juízes escolhidos por e-mail para avaliação inicial quanto à clareza, relevância e objetividade das informações contidas no instrumento. Ao finalizar a avaliação, os juízes enviarão o instrumento com as sugestões de alterações e comentários que julguem necessários. Salienta-se que esta etapa se encontra em andamento no presente momento e a versão finalizada do instrumento será disponibilizada apenas após a avaliação criteriosa feita pelos juízes especialistas e possíveis reformulações necessárias.

\section{Considerações Finais}

O objetivo proposto pelo artigo foi alcançado através da finalização da versão inicial do instrumento de apoio ao processo de enfermagem em Infecções Sexualmente Transmissíveis. Diante da relevância do processo de enfermagem, almejase que o instrumento validado para o atendimento à pessoa com IST possa nortear as ações a serem executadas pelo profissional conforme preconização dos protocolos clínicos nacionais. Sabe-se que a utilização de um instrumento de coleta de dados durante o desenvolvimento da consulta de enfermagem eleva a qualidade da assistência e põe em prática ações de prevenção e controle de IST à toda população, quebrando a rede de transmissão, além de incentivar o julgamento clínico por parte do profissional enfermeiro.

Ressalta-se a relevância da temática abordada, bem como o incentivo de outros estudos que contemplem a abordagem do enfermeiro frente às IST, com o objetivo de ampliar a rede de apoio a esses profissionais. Assim, a partir da construção do instrumento salientou-se a importância de uma consulta de enfermagem completa que aborde os aspectos psicobiológicos, psicossociais e psicoespirituais do paciente, não limitando-se apenas aos dados fisiopatológicos.

Frente a estes aspectos, evidencia-se que esta pesquisa traz importantes contribuições para o avanço da implementação do processo de enfermagem, estimulando o raciocínio clínico e a execução das práticas baseadas em evidências científicas direcionadas a saúde do paciente acometido por IST. Observou-se a necessidade da realização de novos estudos que possibilitem a troca de saberes e detalhe a temática abordada, a fim de incentivar a sistematização da assistência de enfermagem através da sensibilização dos profissionais da área.

\section{Referências}

Almeida, V. S., Querido, D. L \& Vigo, P. S. (2018). Validação de instrumento para histórico de enfermagem materno-infantil utilizando Horta: estudo metodológico. Online Brazilian Journal of Nursing. http://www.objnursing.uff.br/index.php/nursing/article/view/5858/html_2\#: :text=Baseada\%20na\%2 0teoria $\% 2$ de $\% 2$ 0Wanda,de\%20Jo\%C3\%A3o\%20Mohana(2)\%3A.

Araújo, D. S., França, A. F., Mendonça, J. K. S., Bettencourt, A. R. C., Amaral, T. L. M., \& Prado, P. R. (2015). Construção e validação de instrumento de sistematização da assistência de enfermagem em terapia intensiva. Revista Rene. http://www.periodicos.ufc.br/rene/article/view/2737/2121.

Coluci, M. Z. O., Alexandre, N. M. C., Milani \& Milani, D. (2013). Construção de instrumentos de medida na área da saúde. Revista Ciência \& Saúde Coletiva. 10.1590/1413-81232015203.04332013

Barros, A. L. B. L., Sanches, C. G \& Dell'acqua, J. L (2015). Processo de enfermagem: guia para a prática. Conselho Regional de Enfermagem de São Paulo. https://portal.coren-sp.gov.br/sites/default/files/SAE-web.pdf. 
Brasil (2020). Protocolo Clínico e Diretrizes Terapêuticas para Atenção Integral às Pessoas com Infecções Sexualmente Transmissíveis (IST). Ministério da Saúde.

Brasil (2009). Resolução No 358 do Conselho Federal de Enfermagem, de 15 de outubro de 2009. Sistematização da Assistência de Enfermagem e a implementação do Processo de Enfermagem. Ministério da Saúde.

Brasil (2010). Sociedade Brasileira de Medicina de Família e Comunidade - SBMFC. Classificação Internacional de Atenção Primária (CIAP 2).

Brasil (1986). Lei No 7.498/86, de 25 de junho de 1986. Regulamentação do exercício da Enfermagem. http://www.cofen.gov.br/lei-n-749886-de-25de-junho-de-1986_4161.html.

Carvalho, L. O. R., et. al. (2019). Metodologia científica: teoria e aplicação na educação a distância. Fundação Universidade Federal do Vale do São Francisco, Petrolina, Pernambuco.

Coren (2016). Conselho Regional de Enfermagem do Rio Grande do Sul. Parecer Técnico no 19/2016. https://www.portalcorenrs.gov.br/docs/Legislacoes/legislacao_5b25db5b0fcd21efbe3ba956db6e4211.pdf.

Filgueiras, T. F., Silva, R. A., \& Pimenta, C. J. L. (2019). Instrumento para consulta de enfermagem a gestantes com diabetes mellitus. Rev Rene. 10.15253/21756783.20192040104.

Fernandes, R. A. Q., \& Narchi, N. Z (2013). Enfermagem e saúde da mulher. (2a ed.), Manole.

Ferreira, I. T., Neves, K. T. Q. \& Oliveira, A. W. N. (2018). Avaliação da qualidade da consulta de enfermagem em infecções sexualmente transmissíveis. Revista Enfermagem em Foco, Bahia. http://revista.cofen.gov.br/index.php/enfermagem/article/view/1119/459.

Garcia, T. R (2020). Classificação Internacional para a Prática de Enfermagem CIPE(R). Artmed.

Guedes, D. S. (2018). Construção e validação de instrumento para consulta de enfermagem às pessoas com infecções sexualmente transmissíveis (Dissertação de Mestrado). Faculdade de Farmácia, Odontologia e Enfermagem, Universidade Federal do Ceará, Fortaleza. http://www.repositorio.ufc.br/handle/riufc/31695

Gerk, M. A. S., \& Barros, S. M. O. (2005). Intervenções de enfermagem para os diagnósticos de enfermagem mais frequentes em dois serviços públicos de assistência à saúde da mulher. Revista Acta Paul Enferm. https://www.nescon.medicina.ufmg.br/biblioteca/imagem/0745.pdf.

Horta, W. A. (1979). Processo de Enfermagem. E.P.U.

Koerich, M. S., Backes, D. S. \& Scortegagna, H. M. (2007). Tecnologias de cuidado em saúde e enfermagem e suas perspectivas filosóficas. Revista Texto Contexto Enfermagem, https://www.scielo.br/pdf/tce/v15nspe/v15nspea22.pdf.

Polit, D. F.; Beck, C.T. \& Hungler, B. P. (2004). Fundamentos de pesquisa em enfermagem: métodos, avaliação e utilização. 5a Ed. Porto Alegre - RS: Artmed.

Potter, P., \& Perry, A. (2005). Fundamentos de enfermagem. (5a ed.), Guanabara Koogan.

Pereira A. S. et al. (2018). Metodologia da pesquisa científica. UFSM.

Ribeiro, G. C., \& Padoveze, M. C. (2018). Sistematização da Assistência de Enfermagem em unidade básica de saúde: percepção da equipe de enfermagem. Revista da Escola de Enfermagem da USP. https://doi.org/10.1590/s1980-220x2017028803375.

Santos, N., Veiga, P., \& Andrade, R. (2011). Importância da anamnese e do exame físico para o cuidado do enfermeiro. Rev. Bras. Enferm. http://www.scielo.br/scielo.php?script=sci_arttext\&pid=S0034-71672011000200021\&lng=en. http://dx.doi.org/10.1590/S0034-7167201100020002

Santos, S. M. P., Freitas, J. L. G. S. \& Freitas, M. I. F. (2019). Roteiros de sexualidade construídos por enfermeiros e a interface com a atenção em infecções sexualmente transmissíveis/HIV. Esc. Anna Nery. https://doi.org/10.1590/2177-9465-ean-2019-0078en.

Souza Neto, V. L., Silva, R. A. R. \& Rocha, C. C. T. (2017). Diagnósticos de enfermagem da CIPE para pessoas com Síndrome da Imunodeficiência Adquirida. Revista Acta Paul Enferm, https://doi.org/10.1590/1982-0194201700081.

Tolentino, G. S., Bettencourt, A. R. C., \& Fonseca, S. M. (2018). Construção e validação de instrumento para consulta de enfermagem em quimioterapia ambulatorial. Revista Brasileira de Enfermagem. http://dx.doi.org/10.1590/0034-7167-2018-0031.

Truppell, T. C. (2009). Sistematização da Assistência de Enfermagem em Unidade de Terapia Intensiva. Revista Brasileira de Enfermagem. https://www.scielo.br/pdf/reben/v62n2/a08v62n2.pdf.

Viana, V. O., \& Pires, P. S. (2014). Validação de instrumento de sistematização da assistência de enfermagem. Revista Enfermagem Atenção Saúde. http://seer.uftm.edu.br/revistaeletronica/index.php/enfer/article/view/1021/884.

World Health Organization (Who) (2016). Global health sector strategy on sexually transmitted infections, 2016-2021: Towards ending STIs. 